\title{
Repeat Codes, Even Codes, Odd Codes and
}

\section{Their Equivalence}

\author{
Mustafa Özkan and Figen Öke \\ Department of Mathematics, Trakya University, Edirne ,Turkey \\ E-mail: mustafaozkan@trakya.edu.tr \\ Department of Mathematics, Trakya University, Edirne ,Turkey \\ E-mail: figenoke@trakya.edu.tr
}

\begin{abstract}
Codes over the chain ring are obtained by writing special matrices. Gray images of these codes are binary codes. It is shown that first repeat code, second repeat code, even code and odd code are either equivalent or equal to these codes. The definitions of direct sum and direct product of these codes were given. Moreover dual codes were classed. Self dual codes and self orthogonal codes were established.
\end{abstract}

Keywords : Codes over rings, Lee distance, Even codes, Odd codes, Dual codes.

2000 MSC No: 94B15, 94B60.

\section{Introduction}

Different codes over the ring $\mathbb{F}_{2}+u \mathbb{F}_{2}$ with $u^{2}=0$ were studied before. Generally the relation between the codes over $\mathbb{F}_{2}+u \mathbb{F}_{2}$ and binary codes were established. Some of these studies ; $(1+u)$ - cyclic and cyclic codes over the ring $\mathbb{F}_{2}+u \mathbb{F}_{2}$ were studied by J.F.Qian, L.N.Zhang and S.X Zhu in [3]. Some results on cyclic codes over $\mathbb{F}_{2}+v \mathbb{F}_{2}$ were studied by S. Zhu, Y. Wang and M. Shi in [9]. A relation between Hadamard codes and some special codes over $\mathbb{F}_{2}+u \mathbb{F}_{2}$ were studied by $M$. Özkan and F. Öke in [1]. This last study,which was written by forming special matrices over the ring is the Pioneer reference for emergance of this article. In this study, codes over the ring $\mathbb{F}_{2}+u \mathbb{F}_{2}$ are written by using special matrix. Special types of Hadamard codes ara discussed finding binary of these codes.

In the second section, basic code structure was described as weigth function on the ring $\mathbb{F}_{2}+u \mathbb{F}_{2}$ and information about Hadamard codes were given. Gray map was defined. Matrices $N^{\alpha_{1}, \alpha_{2}}$ were formed 
by using elements of the ring $\mathbb{F}_{2}+u \mathbb{F}_{2}$. Then codes $C^{\alpha_{1}, \alpha_{2}}$ were defined with the help of these matrices. It is shown that Gray images of the codes $C^{\alpha_{1}, \alpha_{2}}$ are equal to the Hadamard codes. By this way it is shown that Hadamard codes could be obtained by without using Hadamard matrices.

In the third section, the definition of new code were made with the help of the codes $C^{\alpha_{1}, \alpha_{2}}$. The classification of the new codes were made. It is determined whether the are equivalent orequal to the code $C^{\alpha_{1}, \alpha_{2}}$. Also the definition of odd code an deven code is given fort he codes $C^{\alpha_{1}, \alpha_{2}}$. An important proposition about Gray map an even codes are written.

In the last section, the results about direct sum and direct product of these codes were given. Moreover looking duals of codes and their Gray images the classification is made whether they are self dua lor self ortagonal.

\section{Formulations}

It is known that $\mathbb{F}_{2}+u \mathbb{F}_{2}=\{0,1, u, 1+u\}$ with $u^{2}=0$ is a ring with the usual addition and multiplication. Also it is known that this ring is isomorphic to the ring $\mathbb{F}_{2}[u] /<u^{2}>$ where $u^{2}=0$. The ring $R=\mathbb{F}_{2}+u \mathbb{F}_{2}$ has ideals satisfied the inclusions; $\langle 0\rangle \subseteq\langle u\rangle \subseteq\langle 1\rangle=R$. The $R$ ring is finite chain ring. Let $C$ be a $(n, M, d)_{-}$code. It means that $C$ has the length $n$, it has $M$ elements and it's minimum distance is $d$.

The Lee weight of each $r \in R$ is defined as;

$$
w_{L}(r)= \begin{cases}0 & ; r=0 \\ 1 & ; r=1,1+u . \\ 2 & ; r=u\end{cases}
$$

Then $w_{L}(r)=\sum_{i=1}^{n} w_{L}\left(r_{i}\right)$ is satisfied for each element $r=\left(r_{1}, r_{2}, \ldots, r_{n}\right) \in R^{n}$.

The Hamming weight on $\mathbb{F}_{2}$ is defined as;

$$
w_{H}(c)=\left\{\begin{array}{cc}
0 & ; c=0 \\
1 & ; c=1
\end{array} .\right.
$$

Hence $w_{H}(c)=\sum_{i=1}^{n} w_{H}\left(c_{i}\right)$ is hold for each $c=\left(c_{1}, c_{2}, \ldots, c_{n}\right) \in \mathbb{F}_{2}^{n}$.

The minimum distance of a code $C$ is defined as ;

$d_{L}(C)=\min \left\{d_{L}(x, y)\right\}$, here $x, y \in C, x \neq y$ if $C$ is a code over $R$ and

$d_{H}(C)=\min \left\{d_{H}(x, y)\right\}$, here $x, y \in C, x \neq y$ if $C$ is a code over $\mathbb{F}_{2}$.

Generally the Gray map is defined as :

$$
\begin{aligned}
\Phi: R^{n} & \longrightarrow \mathbb{F}_{2}^{2 n} \\
\left(r_{1}, r_{2}, \ldots, r_{n}\right) & \mapsto\left(b_{1}, b_{2}, \ldots, b_{n}, a_{1}+b_{1}, a_{2}+b_{2}, \ldots, a_{n}+b_{n}\right)
\end{aligned}
$$

where $r_{i}=a_{i}+u b_{i} \in R$ for $1 \leq i \leq n$. 
Therefore $C$ is a code over $\mathbb{F}_{2}+u \mathbb{F}_{2}$ which has length $n$, it's image $\Phi(C)$ under the Gray map will be a binary code which has length $2 n$.

It is clearly seen that the equalities $w_{L}(r)=w_{H}(\Phi(c))$ for each $r \in R^{n}$ are satisfied. Therefore it means that $\Phi$ is an isometry from $\left(R^{n}, d_{L}\right)$ to $\left(\mathbb{F}_{2}^{2 n}, d_{H}\right)$.

A $n \times n$ matrix such that all components are -1 or 1 and $M . M^{t}=n . I$ is called Hadamard matrix. A $n \times n$ matrix is called binary normalized Hadamard matrix if it is obtained from $M_{n} n \times n$ normalized Hadamard matrix writing 0 instead of 1 and writing 1 instead of -1 . Let $A_{n}$ be binary normalized Hadamard matrix of a binary Hadamard matrix $M_{n}$. If each two rows of $A_{n}$ are orthogonal then $\frac{n}{2}$ elements are different for these rows of $A_{n}$.

Think that each row of $A_{n}$ is a vector. Then it is seen that the distance of between two rows is $\frac{n}{2}$. Write each row of matrix as a vector which has $n$ length. Adding themselves and their complements to back of these vectors respectively, new vectors which has $2 n$ length are obtained. Write these new vectors as a code words. If completions of these code words join to this set, it is obtained that a Hadamard code included $4 n$ elements. Thus the minimum distance of this code is $n$.

Choose that all elements of first row of the matrix $N^{\alpha_{1}, \alpha_{2}}$ from the set $\{1\}$, choose that the elements of the other row from the set $\{0,1, u, 1+u\}$ if $\alpha_{2}=0$ and from the set $\{0, u\}$ if $\alpha_{1}=0$. Assume that colums of this matrix are lexicographically ordered. This matrix constructed above is a special matrix which has $\alpha_{1}+\alpha_{2}+1$ rows.

Certain examples for the matrix $N^{\alpha_{1}, \alpha_{2}}$ constructed above are given below :

$$
\begin{aligned}
& N^{0,0}=[1], N^{0,1}=\left[\begin{array}{ll}
1 & 1 \\
0 & u
\end{array}\right], N^{0,2}=\left[\begin{array}{cccc}
1 & 1 & 1 & 1 \\
0 & 0 & u & u \\
0 & u & 0 & u
\end{array}\right], N^{0,3}=\left[\begin{array}{cccccccc}
1 & 1 & 1 & 1 & 1 & 1 & 1 & 1 \\
0 & 0 & 0 & 0 & u & u & u & u \\
0 & 0 & u & u & 0 & 0 & u & u \\
0 & u & 0 & u & 0 & u & 0 & u
\end{array}\right] \text {, } \\
& N^{1,0}=\left[\begin{array}{cccc}
1 & 1 & 1 & 1 \\
0 & 1 & u & 1+u
\end{array}\right], N^{2,0}=\left[\begin{array}{cccccccccccccccc}
1 & 1 & 1 & 1 & 1 & 1 & 1 & 1 & 1 & 1 & 1 & 1 & 1 & 1 & 1 & 1 \\
0 & 0 & 0 & 0 & 1 & 1 & 1 & 1 & u & u & u & u & 1+u & 1+u & 1+u & 1+u \\
0 & 1 & u & 1+u & 0 & 1 & u & 1+u & 0 & 1 & u & 1+u & 0 & 1 & u & 1+u
\end{array}\right], \\
& N^{1,1}=\left[\begin{array}{cccccccc}
1 & 1 & 1 & 1 & 1 & 1 & 1 & 1 \\
0 & 0 & 1 & 1 & u & u & 1+u & 1+u \\
0 & u & 0 & u & 0 & u & 0 & u
\end{array}\right]
\end{aligned}
$$

Define the code $C^{\alpha_{1}, \alpha_{2}}=\left\{\left(c_{1}, c_{2}\right) \cdot N^{\alpha_{1}, \alpha_{2}} \mid c_{1} \in R^{\alpha_{1}+1}, c_{2} \in \mathbb{F}_{2}^{\alpha_{2}}\right\}$ which has a generator matrix $N^{\alpha_{1}, \alpha_{2}}$ where $\alpha_{1}, \alpha_{2}$ are inregers such that $\alpha_{1}, \alpha_{2} \geq 0$. The lenght of this code is $n=2^{2 \alpha_{1}+\alpha_{2}}$. Moreover the parameter of the code $C^{\alpha_{1}, \alpha_{2}}$ over $\mathbb{F}_{2}+u \mathbb{F}_{2}$ are $(n, 4 n, n)$. If $C^{\alpha_{1}, \alpha_{2}}$ is a code generated by the matrix $N^{\alpha_{1}, \alpha_{2}}$ over $\mathbb{F}_{2}+u \mathbb{F}_{2}$, it's image $\Phi\left(C^{\alpha_{1}, \alpha_{2}}\right)$ under the Gray map is the $(2 n, 4 n, n)$ _ Hadamard code over the field $\mathbb{F}_{2}$.

The generating matrices of the $\Phi\left(C^{\alpha_{1}, \alpha_{2}}\right)$ codes over $\mathbb{F}_{2}$ are $M$ Hadamard matrices. 


\section{Odd codes, even codes and recurrent construction codes of the code $C^{\alpha_{1}, \alpha_{2}}$}

Let $n=2^{2 \alpha_{1}+\alpha_{2}}$ where $\alpha_{1}, \alpha_{2} \geq 0$. It is known that $C^{\alpha_{1}, \alpha_{2}}=\left\{\left(c_{1}, c_{2}\right) \cdot N^{\alpha_{1}, \alpha_{2}} \mid c_{1} \in R^{\alpha_{1}+1}, c_{2} \in \mathbb{F}_{2}^{\alpha_{2}}\right\}$ is a $(n, 4 n, n)$-code over the ring $R$. Hence $S^{\prime}=\{00 \ldots 0, u u \ldots u\}$ is a $(n, 2,2 n)$-code and $S^{\prime \prime}=\{00 \ldots 0,11 \ldots 1, u u \ldots u, 1+u 1+u \ldots 1+u\}$ is a $(n, 4, n)$ - code over the ring $R$.

Definition 3.1 The codes ${ }_{1} C^{\alpha_{1}, \alpha_{2}}=\left\{(a, a+b) \mid a \in C^{\alpha_{1}, \alpha_{2}}, b \in S^{\prime}\right\}$ and ${ }_{2} C^{\alpha_{1}, \alpha_{2}}=\left\{(a, a+b, a+u b, a+(1+u) b) \mid a \in C^{\alpha_{1}, \alpha_{2}}, b \in S^{\prime \prime}\right\}$ is defined by using the codes $S^{\prime}, S^{\prime \prime}$ and $C^{\alpha_{1}, \alpha_{2}}$. Let ${ }_{1} C^{\alpha_{1}, \alpha_{2}}=\left\{(a, a+b) \mid a \in C^{\alpha_{1}, \alpha_{2}}, b \in S^{\prime}\right\}$ and ${ }_{2} C^{\alpha_{1}, \alpha_{2}}=\left\{(a, a+b, a+u \cdot b, a+(1+u) \cdot b) \mid a \in C^{\alpha_{1}, \alpha_{2}}, b \in S^{\prime \prime}\right\}$ where $S^{\prime}, S^{\prime \prime}$ and $C^{\alpha_{1}, \alpha_{2}}$ are defined as above ${ }_{1} C^{\alpha_{1}, \alpha_{2}}$ is called first repeat code over $R$ which has parameters $(2 n, 8 n, 2 n) \cdot{ }_{2} C^{\alpha_{1}, \alpha_{2}}$ is called second repeat code over $R$ which has parameters $(4 n, 16 n, 4 n)$.

Preposition 3.2 The code ${ }_{1} C^{\alpha_{1}, \alpha_{2}}$ is equivalent to the code $C^{\alpha_{1}, \alpha_{2}+1}$. Specially ${ }_{1} C^{0, \alpha_{2}}=C^{0, \alpha_{2}+1}$ is satisfied.

Proof. Here $b=00 \ldots 0$ or $b=u u . . . u$ fort he code ${ }_{1} C^{\alpha_{1}, \alpha_{2}}$.

For each codeword $a \in C^{\alpha_{1}, \alpha_{2}}$ with length $n=2^{2 \alpha_{1}+\alpha_{2}}$ then for each codeword $x=(a, a+b) \in_{1} C^{\alpha_{1}, \alpha_{2}}$ has length $2 n$. Thus $2 n=2.2^{2 \alpha_{1}+\alpha_{2}}=2^{2 \alpha_{1}+\left(\alpha_{2}+1\right)}$ is satisfied. Similarly the number of elements of the code $C^{\alpha_{1}, \alpha_{2}}$ will be two times of the number of elements of $S^{\prime} . d_{L}(a)=n, d_{L}(a+b)=n$ since $d_{L}(b)=0$ or $d_{L}(b)=2 n$. Then $d_{L}(x)=2 n$ for each $x \in \in_{1} C^{\alpha_{1}, \alpha_{2}}$ so the code which has length $2^{2 \alpha_{1}+\left(\alpha_{2}+1\right)}$ is equivalent the code $C^{\alpha_{1}, \alpha_{2}}=\left\{\left(c_{1}, c_{2}\right) \cdot N^{\alpha_{1}, \alpha_{2}+1} \mid c_{1} \in R^{\alpha_{1}+1}, c_{2} \in \mathbb{F}_{2}^{\alpha_{2}+1}\right\}$. Especially in the case $\alpha_{1}=0$, each rows of the matrix $N^{0, \alpha_{2}}$ in except the first row are consisted of the elements 0 and $u$. It is seen that these codes are $C^{0, \alpha_{2}+1}$

Preposition 3.3 Let $\alpha_{1}, \alpha_{2} \geq 0$, Then the code ${ }_{2} C^{\alpha_{1}, \alpha_{2}}$ is equivalent to the code $C^{\alpha_{1}+1, \alpha_{2}}$. ${ }_{2} C^{\alpha_{1}, \alpha_{2}}$ is equal tho the code $C^{\alpha_{1}+1, \alpha_{2}}$ when $\alpha_{1}=0$.

Proof. Let the length of $C^{\alpha_{1}, \alpha_{2}}$ be $n$. The length of the code ${ }_{2} C^{\alpha_{1}, \alpha_{2}}$ is $4 n=2^{2} \cdot 2^{2 \alpha_{1}+\alpha_{2}}=2^{2\left(\alpha_{1}+1\right)+\alpha_{2}}$ since the length of the code ${ }_{2} C^{\alpha_{1}, \alpha_{2}}$ is 4 times of the length of the code $C^{\alpha_{1}, \alpha_{2}}$. Proof is similarly completed to the proof of the Preposition 3.2.

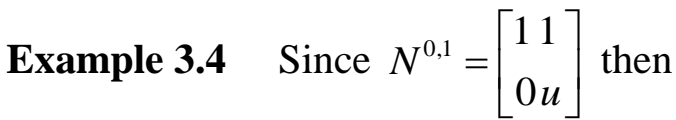
$C^{0,1}=\left\{\left(c_{1}, c_{2}\right) \cdot N^{0,1} \mid c_{1} \in R, c_{2} \in \mathbb{F}_{2}\right\}=\{00,11, u u, 1+u 1+u, 0 u, u 0,11+u, 1+u 1\} \subseteq R^{2}$ is the $(2,8,2) \_$code.

Let $S^{\prime}=\{00, u u\}$. Then ${ }_{1} C^{0,1}=\left\{(a, a+b) \mid a \in C^{0,1}, b \in S^{\prime}\right\}$ 
$=\left\{\begin{array}{c}0000, \text { иии }, 0 u 0 u, u 0 u 0,1111,1+u 1+u 1+u 1+u, 11+u 11+u, 1+u 11+u 1 \\ 00 u u, u u 00,0 u u 0, u 00 u, 111+u 1+u, 1+u 1+u 11,11+u 1+u 1,1+u 111+u\end{array}\right\} \subseteq R^{4}$ is a $(4,16,4) \_$code.

It is seen that this is equal the code $C^{0,2}$ obtained by the matrix $N^{0,2}$

The code ${ }_{2} C^{0,1}=\left\{(a, a+b, a+u b, a+(1+u), b) \mid a \in C^{0,1}, b \in S^{\prime \prime}\right\}$ cis equal to the code $C^{1,1}$ generated by the matrix $N^{1,1}$. The parameter of the code ${ }_{2} C^{0,1}$ is $(8,32,8)$.

Example 3.5 Writing the code

$C^{1,0}=\{0000,1111$, ии u $u, 1+u 1+u 1+u 1+u, 01 u 1+u, 101+u u, u 1+u 01,1+u u 10$,

$0 u 0 u, 11+u 11+u, u 0 u 0,1+u 11+u 1,01+u u 1,1 u 1+u 0, u 101+u, 1+u 01 u\}^{\text {with the }}$

parameter $(4,16,4)$, the code

${ }_{1} C^{1,0}=\{00000000,0000 u u u u, 01 u 1+u 01 u 1+u, 01 u 1+u u 1+u 01$,

$0 u 0 u 0 u 0 u, 0 u 0 u u 0 u 0,01+u u 101+u u 1,01+u u 1 u 101+u$,

$11111111,11111+u 1+u 1+u 1+u, 101+u u 101+u u, 101+u u 1+u u 10$,

$1+u 1+u 1+u 1+u 1+u 1+u 1+u 1+u, 1+u 1+u 1+u 1+u 1111,1+u u 101+u u 10,1+u u 10101+u u, \quad$ is

$11+u 11+u 11+u 11+u, 11+u 11+u 1+u 11+u 1,1 u 1+u 01 u 1+u 0,1 u 1+u 01+u 01 u$,

$1+u 11+u 11+u 11+u 1,1+u 11+u 111+u 11+u, 1+u 01 u 1+u 01 u, 1+u 01 u 1 u 1+u 0$,

ииииииии, $u 0 u 0 u 0 u 0, u и 1+u 1+u 0011, u 01+u 10 u 11+u$,

$u и 00 u u 00, u 00 u u 00 u, u u 11001+u 1+u, u 011+u 0 u 1+u 1\} \subseteq R^{8}$

obrained. Hence, using a suitable rotation and permutation the components of ${ }_{1} C^{1,0}$, the code $C^{1,1}$ is obtained. Then it is seen that the code ${ }_{1} C^{1,0}$ is equivalent to the code $C^{1,1}$. Moreover it is seen that, the code ${ }_{2} C^{1,0}$ is equivalent to the code $C^{2,0}$.

Definition 3.6 Let $C^{\alpha_{1}, \alpha_{2}} \subseteq R^{n}$ be a code.

$\operatorname{even}\left(C^{\alpha_{1}, \alpha_{2}}\right)=\left\{\left(c_{0}, c_{2}, \ldots, c_{n-2}\right) \in R^{\frac{n}{2}} \mid\left(c_{0}, c_{1}, \ldots, c_{n-1}\right) \in C^{\alpha_{1}, \alpha_{2}}\right\}$ is called an even code over $R^{\frac{n}{2}}$.

$\operatorname{odd}\left(C^{\alpha_{1}, \alpha_{2}}\right)=\left\{\left(c_{1}, c_{3}, \ldots, c_{n-1}\right) \in R^{\frac{n}{2}} \mid\left(c_{0}, c_{1}, \ldots, c_{n-1}\right) \in C^{\alpha_{1}, \alpha_{2}}\right\}$ is called an odd code over $R^{\frac{n}{2}}$. Even codes and odd codes are defined over the field $\mathbb{F}_{2}$ writing $\mathbb{F}_{2}$ instead of $R$.

Preposition 3.7 Let $C^{\alpha_{1}, \alpha_{2}}$ be a code.

i) $\operatorname{even}\left(C^{\alpha_{1}, \alpha_{2}}\right)=\operatorname{odd}\left(C^{\alpha_{1}, \alpha_{2}}\right)=C^{\alpha_{1}-1, \alpha_{2}+1}$ is satisfied if $\alpha_{1} \geq 1, \alpha_{2} \geq 0$.

ii) $\operatorname{even}\left(C^{\alpha_{1}, \alpha_{2}}\right) \approx \operatorname{odd}\left(C^{\alpha_{1}, \alpha_{2}}\right)=C^{\alpha_{1}, \alpha_{2}-1}$ is satisfied if $\alpha_{1} \geq 0, \alpha_{2} \geq 1$.

Preposition 3.8 even $\left(\Phi\left(C^{\alpha_{1}, \alpha_{2}}\right)\right)=\Phi\left(\operatorname{even}\left(C^{\alpha_{1}, \alpha_{2}}\right)\right)$ is satisfied.

Proof. Let $c=\left(c_{0}, c_{1}, \ldots, c_{n-1}\right) \in C^{\alpha_{1}, \alpha_{2}}$ where $c_{i}=r_{i}+u q_{i}$ for $0 \leq i \leq n$.

If $\Phi(c)=\Phi\left(c_{0}, c_{1}, \ldots, c_{n-1}\right)=\Phi\left(r_{0}+u q_{0}, r_{1}+u q_{1}, \ldots, r_{n-1}+u q_{n-1}\right)$

$$
=\left(q_{0}, q_{1}, \ldots, q_{n-1}, r_{0}+q_{0}, r_{1}+q_{1}, \ldots, r_{n-1}+q_{n-1}\right) \in \Phi\left(C^{\alpha_{1}, \alpha_{2}}\right)
$$

then $\left(q_{0}, q_{2}, \ldots, q_{n-2}, r_{0}+q_{0}, r_{2}+q_{2}, \ldots, r_{n-2}+q_{n-2}\right) \in \operatorname{even}\left(\Phi\left(C^{\alpha_{1}, \alpha_{2}}\right)\right)$.

On the other hand, 
$c^{\prime}=\left(c_{0}, c_{2}, \ldots, c_{n-2}\right)=\left(r_{0}+u q_{0}, r_{2}+u q_{2}, \ldots, r_{n-2}+u q_{n-2}\right) \in \operatorname{even}\left(C^{\alpha_{1}, \alpha_{2}}\right)$. Then

$\Phi\left(c^{\prime}\right)=\left(q_{0}, q_{2}, \ldots, q_{n-2}, r_{0}+q_{0}, r_{2}+q_{2}, \ldots, r_{n-2}+q_{n-2}\right) \in \Phi\left(\operatorname{even}\left(C^{\alpha_{1}, \alpha_{2}}\right)\right)$.

This Preposition can be written fort he odd codes.

Example 3.9 For the code

$C^{0,2}=\{0000,0 u 0 u, 00 u u, 0 u u 0,1111,11+u 11+u, 111+u 1+u, 11+u 1+u 1$, ииии,

$u 0 u 0, u u 00, u 00 u, 1+u 1+u 1+u 1+u, 1+u 11+u 1,1+u 1+u 11,1+u 111+u\} \subseteq R^{4}$,

is obtained. even $\left(C^{0,2}\right)=\{00, u 0,0 u, u u, 11,11+u, 1+u 1,1+u 1+u\}$ is obtained.

$\Phi\left(\operatorname{even}\left(C^{0,2}\right)\right)=\{0000,0011,1111,1100,0101,0110,1010,1001\}$ and

$\Phi\left(C^{0,2}\right)=\{00000000,01010101,00110011,01100110,00001111,01011010,00111100,01101001$,

$11111111,10101010,11001100,10011001,11110000,10100101,11000011,10010110\}$

Hence even $\left(\Phi\left(C^{0,2}\right)\right)=\Phi\left(\right.$ even $\left.\left(C^{0,2}\right)\right)$ is obtained.

Example 3.10 The even code of $C^{1,0}$ given in the example 3.5. is $\operatorname{even}\left(C^{1,0}\right)=\{00, u 0,11,11+u, u u, u 0,1+u 1+u, 1+u 1\}=\operatorname{odd}\left(C^{1,0}\right)=C^{0,1}$.

Example 3.11 Let $C^{1,1}=\left\{\left(c_{1}, c_{2}\right) \cdot N^{1,1} \mid c_{1} \in R^{2}, c_{2} \in \mathbb{F}_{2}\right\}$ with the parameter $(8,32,8)$.

$\operatorname{even}\left(C^{1,1}\right)=\{0000,0 u 0 u, 00 u u, 0 u u 0,1111,11+u 11+u, 111+u 1+u, 11+u 1+u 1$, $1+u 1+u 1+u 1+u, 1+u 11+u 1,1+u 1+u 11,1+u 111+u, u u u u, u 0 u 0, u 101+u, u 1+u 01\} \subseteq R^{4}$.

e parameter of this code is $(4,16,4)$. Using a suitable rotation and permutation to the components of this code the code

$C^{1,0}=\{0000,1111, u u u u, 1+u 1+u 1+u 1+u, 01 u 1+u, 101+u u, u 1+u 01,1+u u 10$,

$0 u 0 u, 11+u 11+u, u 0 u 0,1+u 11+u 1,01+u u 1,1 u 1+u 0, u 101+u, 1+u 01 u\}$ is obtained.

It was shown that the Gray images of the codes $C^{\alpha_{1}, \alpha_{2}}$ are Hadamard codes in [1]. In this sstudy; it is shown that ${ }_{1} C^{\alpha_{1}, \alpha_{2}},{ }_{2} C^{\alpha_{1}, \alpha_{2}}, \operatorname{even}\left(C^{\alpha_{1}, \alpha_{2}}\right)$ and $\operatorname{odd}\left(C^{\alpha_{1}, \alpha_{2}}\right)$ are either equivalent or equal to the code $C^{\alpha_{1}, \alpha_{2}}$ and so these codes are Hadamard codes.

\section{The stucture of the linear code over $\mathbb{F}_{2}+u \mathbb{F}_{2}$}

Let $A, B \subseteq R^{n}$. It is written that $A \otimes B=\{(a, b) \mid a \in A, b \in B\}$ and $A \oplus B=\{a+b \mid a \in A, b \in B\}$. Let $C^{\alpha_{1}, \alpha_{2}}$ be a code with length $n$ over $R$. Define $C_{1}^{\alpha_{1}, \alpha_{2}}=\left\{x \in \mathbb{F}_{2}^{n} \mid \exists y \in \mathbb{F}_{2} \ni x+u y \in C^{\alpha_{1}, \alpha_{2}}\right\}$ and $C_{2}^{\alpha_{1}, \alpha_{2}}=\left\{x+y \in \mathbb{F}_{2}^{n} \mid x+u y \in C^{\alpha_{1}, \alpha_{2}}\right\} . C_{1}^{\alpha_{1}, \alpha_{2}}, C_{2}^{\alpha_{1}, \alpha_{2}}$ are binary codes. 
Preposition 4.1 $C^{\alpha_{1}, \alpha_{2}+1} \subseteq C^{\alpha_{1}, \alpha_{2}} \otimes C^{\alpha_{1}, \alpha_{2}}$ is satisfied.

Proof. Take $c=\left(c_{0}, c_{1}, \ldots, c_{n-1}\right) \in C^{\alpha_{1}, \alpha_{2}+1}$. The length of the code $C^{\alpha_{1}, \alpha_{2}+1} \subseteq R^{n}$ is $n=2^{2 \alpha_{1}+\alpha_{2}+1}$. For each codeword $c=\left(c_{0}, c_{1}, \ldots, c_{n-1}\right)=\left(c_{0}, c_{1}, \ldots, c_{2^{2 \alpha_{1}+\alpha_{2}+1}}\right)=\left(c_{0}, c_{1}, \ldots, c_{2^{2 \alpha_{1}+\alpha_{2}}}, c_{2^{2 \alpha_{1}+\alpha_{2}}+1}, \ldots, c_{2^{2 \alpha_{1}+\alpha_{2}+1}}\right) \in C^{\alpha_{1}, \alpha_{2}} \otimes C^{\alpha_{1}, \alpha_{2}}$ is satisfied.

Theorem 4.2 Let $C^{\alpha_{1}, \alpha_{2}}$ be a code of length $n=2^{2 \alpha_{1}+\alpha_{2}}$ over $R$. Then $C_{1}^{\alpha_{1}, \alpha_{2}} \otimes C_{2}^{\alpha_{1}, \alpha_{2}} \approx \Phi\left(C^{\alpha_{1}, \alpha_{2}}\right)$ and $\left|C^{\alpha_{1}, \alpha_{2}}\right|=\left|C_{1}\right| \cdot\left|C_{2}\right|$.

Proof. For each $\left(r_{1}, r_{2}, \ldots, r_{n}, q_{1}+r_{1}, q_{2}+r_{2}, \ldots, q_{n}+r_{n}\right)$ is element of $\Phi\left(C^{\alpha_{1}, \alpha_{2}}\right)$. Let $c_{i}=r_{i}+u q_{i}$, $i=1, \ldots, n . c=\left(c_{1}, c_{2}, \ldots, c_{n}\right) \in C^{\alpha_{1}, \alpha_{2}}$ since $\Phi$ is a bijection. Using the definitions of $C_{1}^{\alpha_{1}, \alpha_{2}}$ and $C_{2}^{\alpha_{1}, \alpha_{2}}$, we obtained that $\left(r_{1}, r_{2}, \ldots, r_{n}\right) \in C_{1}^{\alpha_{1}, \alpha_{2}},\left(q_{1}+r_{1}, q_{2}+r_{2}, \ldots, q_{n}+r_{n}\right) \in C_{2}^{\alpha_{1}, \alpha_{2}}$,thus $\left(r_{1}, r_{2}, \ldots, r_{n}, q_{1}+r_{1}, q_{2}+r_{2}, \ldots, q_{n}+r_{n}\right) \in C_{1}^{\alpha_{1}, \alpha_{2}} \otimes C_{2}^{\alpha_{1}, \alpha_{2}}$. This implies that $\Phi\left(C^{\alpha_{1}, \alpha_{2}}\right) \subseteq C_{1}^{\alpha_{1}, \alpha_{2}} \otimes C_{2}^{\alpha_{1}, \alpha_{2}}$. $C_{1}^{\alpha_{1}, \alpha_{2}} \otimes C_{2}^{\alpha_{1}, \alpha_{2}} \subseteq \Phi\left(C^{\alpha_{1}, \alpha_{2}}\right)$ is similarly obtained. This means that all elements of the code $C_{1}^{\alpha_{1}, \alpha_{2}} \otimes C_{2}^{\alpha_{1}, \alpha_{2}}$ are equivalent to the elements of the code $\Phi\left(C^{\alpha_{1}, \alpha_{2}}\right)$. The second result is easy to verify.

Corollary 4.3 If $\Phi\left(C^{\alpha_{1}, \alpha_{2}}\right) \approx C_{1}^{\alpha_{1}, \alpha_{2}} \otimes C_{2}^{\alpha_{1}, \alpha_{2}}$

i) $C^{\alpha_{1}, \alpha_{2}}=C_{1}^{\alpha_{1}, \alpha_{2}} \oplus(u) C_{2}^{\alpha_{1}, \alpha_{2}}$ when $\alpha_{1}=0$

ii) $C^{\alpha_{1}, \alpha_{2}} \subseteq C_{1}^{\alpha_{1}, \alpha_{2}} \oplus(u) C_{2}^{\alpha_{1}, \alpha_{2}}$ when $\alpha_{1} \neq 0$

Preposition 4.4 Let $C^{\alpha_{1}, \alpha_{2}} \subseteq R^{n}$ be a code. $d_{H}=d_{L}=\min \left\{d_{H}\left(C_{1}^{\alpha_{1}, \alpha_{2}}\right), d_{H}\left(C_{2}^{\alpha_{1}, \alpha_{2}}\right)\right\}$ is satisfied.

Proof. It is known that distances are invariant under the map $\Phi . d_{L}\left(C^{\alpha_{1}, \alpha_{2}}\right)=d_{H}\left(\Phi\left(C^{\alpha_{1}, \alpha_{2}}\right)\right)$. Since $\Phi\left(C^{\alpha_{1}, \alpha_{2}}\right) \approx C_{1}^{\alpha_{1}, \alpha_{2}} \otimes C_{2}^{\alpha_{1}, \alpha_{2}} \cdot d_{H}\left(\Phi\left(C^{\alpha_{1}, \alpha_{2}}\right)\right)=d_{H}\left(C_{1}^{\alpha_{1}, \alpha_{2}} \otimes C_{2}^{\alpha_{1}, \alpha_{2}}\right)=\min \left\{d_{H}\left(C_{1}^{\alpha_{1}, \alpha_{2}}\right), d_{H}\left(C_{2}^{\alpha_{1}, \alpha_{2}}\right)\right\}$ is obtained.

Preposition 4.5 The codes are a self orthogonal codes except the codes $C^{0,0}$ and $C^{0,1}$. The codes $C^{1,0}$ and $C^{0,2}$ are self dual codes.

Proof. It is known that code $C^{\alpha_{1}, \alpha_{2}}$ is $n=2^{2 \alpha_{1}+\alpha_{2}}$. The number of elements of the code $\left(C^{\alpha_{1}, \alpha_{2}}\right)^{\perp}$ is $\frac{4^{n}}{4 . n}$ since the number of elements of the code $C^{\alpha_{1}, \alpha_{2}}$ is $4 n$. So $4 n \leq \frac{4^{n}}{4 . n}$ except the cases $\left(\alpha_{1}, \alpha_{2}\right)=(0,0)$ and $\left(\alpha_{1}, \alpha_{2}\right)=(0,1)$. Then $C^{\alpha_{1}, \alpha_{2}} \subseteq\left(C^{\alpha_{1}, \alpha_{2}}\right)^{\perp}$ is satisfied.

Preposition 4.6 The code $\Phi\left(C^{\alpha_{1}, \alpha_{2}}\right)$ a self orthogonal code, except the codes $\Phi\left(C^{0,0}\right)$ and $\Phi\left(C^{0,1}\right)$. Specially the codes $\Phi\left(C^{0,1}\right)$ and $\Phi\left(C^{0, \alpha_{2}}\right)$ are self dual codes. 
Proof. It can be similarly obtained to the proof of Preposition 4.5.

Preposition $4.7\left(C_{1}^{\alpha_{1}, \alpha_{2}}\right)^{\perp}=C_{1}^{\alpha_{1}, \alpha_{2}}$, except the codes $C^{0,0}$ and $C^{0,1}$. Either $\left(C_{2}^{\alpha_{1}, \alpha_{2}}\right)^{\perp}=C_{2}^{\alpha_{1}, \alpha_{2}}$ or $\left(C_{2}^{\alpha_{1}, \alpha_{2}}\right)^{\perp}$ is equal the a repeation code over $\mathbb{F}_{2}$

\section{References}

[1] M. Özkan, F. Öke, " A relation between Hadamard codes and some special codes over $\mathbb{F}_{2}+u \mathbb{F}_{2}$ ", App.Mathematics and Inf Sci, Vol.10, No.2, (2016), pp.701-7047.

[2] J.Wolfmann, "Negacyclic and cyclic codes over $Z_{4}$ ”, IEEE Trans. Inf. Theory, Vol.45, (1999), pp. $2527-2532$

[3] J.F.Qian, L.N.Zhang, S.X Zhu, ” $(1+u)$-constacyclic and cyclic codes over $\mathbb{F}_{2}+u \mathbb{F}_{2}$, Applied Mathematics Letters", Vol.19,(2006),pp.820-823.

[4] Krotov, D. S., "Z4-linear perfect codes", Diskretn. Anal. Issled. Oper. Ser.1.Vol. 7. No.4.(2000),pp.78-90

[5] M. Ozkan, F. Oke, "Codes defined via especial matrices over the ring and Hadamard codes", Mathematical Sciences and Applications E-Notes, Vol.5,No.1 (2017),pp.93-98.

[6] A. Bonnecaze and P. Udaya, "Cyclic codes and self dual codes $\mathbb{F}_{2}+u \mathbb{F}_{2}$ ", IEEE Trans. Inf. Theory,Vol.45, (1999),pp.1250-1255.

[7] M. Özkan, F. Öke, "Some Special Codes Over $\mathbb{F}_{3}+v \mathbb{F}_{3}+u \mathbb{F}_{3}+u^{2} \mathbb{F}_{3}$ " Mathematical Sciences and Applications E-Notes, Vol. 4, No.1, (2016), pp. 40-44.

[8] M. Özkan, F. Öke, "Gray images of $(1+\mathrm{v})$ - constacyclic codes over a particular ring ", Palestine Journal of Mathematics. Vol. 6, No.Si. 2, (2017) pp. 241-245.

[9] S. Zhu, Y. Wang, M. Shi, Some Result On Cylic Codes Over $\mathbb{F}_{2}+v \mathbb{F}_{2}$, IEEE Trans. Inf. Theory, Vol.56, No.4,(2010),pp.1680-1684.

[10] Vermani, L. R., "Elements of Algebraic Coding Theory", Chapman Hall, India., (1996). 